\title{
Transformation of the Stäckel matrices preserving superintegrability
}

\author{
A.V. Tsiganov \\ St.Petersburg State University, St.Petersburg, Russia \\ e-mail: andrey.tsiganov@gmail.com
}

\begin{abstract}
If we take a superintegrable Stäckel system and make variables "faster" or "slower", that is equivalent to a trivial transformation of the Stäckel matrix and potentials, then we obtain an infinite family of superintegrable systems with explicitly defined additional integrals of motion. We present some examples of such transformations associated with angle variables expressed via logarithmic functions.
\end{abstract}

\section{Introduction}

Let us consider a superintegrable system with closed trajectories. Various time parameterizations of the given trajectories can be associated with different superintegrable systems. The relation between harmonic oscillator and Kepler problem is a main example of such time reparametrizations, which are called Jacobi-Maupertuis transformation, canonical transformation of the extended phase space, Stäckel transformation, coupling constant metamorphosis, reciprocal transformation, etc., see [5, 11, 22, 37, 42, 43. The main problem here is how to construct additional integrals of motion associated with different times. The purpose of this paper is to discuss trivial transformation of the Stäckel matrix and potentials which maps the given superintegrable Stäckel system to an infinite family of superintegrable systems with explicitly defined additional integrals of motion.

A Hamiltonian system on a $2 n$-dimensional symplectic manifold $M$ endowed with symplectic form $\Omega$ is said to be completely integrable if it has $n$ smooth functionally independent functions $H_{1}, \ldots, H_{n}$ in involution

$$
\left\{H_{i}, H_{j}\right\}=0, \quad i, j=1, \ldots, n
$$

which define Lagrangian submanifold in a phase space [9, 36.

Integrable system with additional independent integrals of motion $X_{1}, \ldots, X_{k}$ commuting with the Hamiltonian $H=H_{1}$

$$
\left\{H, X_{i}\right\}=0, \quad i=1, \ldots, k,
$$

is the so-called superintegrable system if all the integrals $H_{j}$ and $X_{i}$ are well defined functions on phase space or an open submanifold of phase space.

Below we suppose that trajectories of superintegrable systems lie on an intersection of $n$-dimensional Lagrangian submanifold with $k$ hyperplanes $X_{i}=$ const. There are also many systems with $n+k$ integrals of motion which have motions linear on tori of smaller dimension $n-k$ [9, 36]. We prefer to study an intersection of algebraic varieties because it allows us to apply standard algebraic geometry methods, for instance Abel's theorem, to construction of integrals of motion.

Properties of the Lagrangian submanifold are described by the Liouville theorem, which implies that almost all points of phase space are covered by a system of open toroidal domains 
with the canonical action-angle coordinates $I_{1}, \ldots, I_{n}$ and $\omega_{1}, \ldots, \omega_{n}$ so that

$$
\dot{I}_{j}=0, \quad \dot{\omega}_{j}=\frac{\partial H}{\partial I_{j}}, \quad \Omega=\sum_{j=1}^{n} d I_{j} \wedge \omega_{j}
$$

Of course, in general not the entire phase space of a superintegrable system is fibred by invariant tori of a given dimension $n$ because in fact the orbits form a foliation with singularities. In order to construct additional integrals of motion it is enough to consider the regular subset of phase space $M$ where any function $X$ independent on $H_{1}, \ldots, H_{n}$ has to be some well-defined function on angle variables, see discussion in 31.

In 1837 Jacobi proved that a product of $n$ smooth plane curves $C_{1} \times \cdots \times C_{n}$ is the Lagrangian submanifold in $\mathbb{R}^{2 n}[23,24]$. Indeed, if we take $n$ plane curves $C_{j}$ defined by equations of the form

$$
\Phi_{j}\left(u_{j}, p_{u_{j}}, H_{1}, \ldots, H_{n}\right)=0, \quad j=1, \ldots, n, \quad \operatorname{det}\left[\frac{\partial \Phi_{j}}{\partial H_{k}}\right] \neq 0,
$$

then solutions of these relations with respect to $H_{1}, \ldots, H_{n}$ are in involution according to the Jacobi theorem.

In 1891 Stäckel found action-angle variables

$$
I_{i}=\sum_{j=1}^{n} S_{j i}^{-1}\left(p_{u_{j}}^{2}+V_{j}\left(u_{j}\right)\right), \quad i=1, \ldots, n
$$

and

$$
\omega_{i}=\frac{1}{n} \sum_{j=1}^{n} \int \frac{S_{i j}\left(u_{j}\right)}{\sqrt{\sum_{m=1}^{n} I_{m} S_{m j}\left(u_{j}\right)-V_{j}\left(u_{j}\right)}} \mathrm{d} u_{j}
$$

for the Jacobi integrable systems with Hamiltonians

$$
H=F\left(I_{1}, \ldots, I_{n}\right)
$$

associated with a product $C_{1} \times \cdots \times C_{n}$ of hyperelliptic curves of the form

$$
C_{j}: \quad p_{u_{j}}^{2}=\phi_{j}\left(u_{j}, H_{1}, \ldots, H_{n}\right) \equiv \sum_{k=1}^{n} H_{k} S_{k j}\left(u_{j}\right)-V_{j}\left(u_{j}\right)
$$

Here $S$ is the so-called Stäckel matrix, which $j$ column depends only on coordinate $u_{j}$, and $V_{j}\left(u_{j}\right)$ are Stäckel potentials. These action-angle variables have been reconstructed in many partial cases by using complete solution of the Hamilton-Jacobi equation and the corresponding generating function or by integration of the form $\theta=\sum p_{u_{i}} d u_{i}$ along basic cycles of the corresponding torus, for instance see [12, 13, 25, 31.

Changing first row in the Stäckel matrix

$$
S \rightarrow \tilde{S}, \quad S_{i j}=\tilde{S}_{i j}, \quad i \neq 1
$$

we make the canonical transformation of the extended phase space

$$
H \rightarrow \tilde{H}=v^{-1}(H+c), \quad d t \rightarrow d \tau=v d t,
$$

where

$$
v=\frac{\operatorname{det} S}{\operatorname{det} \tilde{S}},
$$

which always preserves integrability and, sometimes, superintegrability [5, 11, 22, 37, 42, 43.

In this note we study transformations which always preserve superintegrability. Indeed, let us take a superintegrable Stäckel system with independent integral of motion

$$
X=F\left(\omega_{1}, \omega_{2}, \ldots, \omega_{n}\right) .
$$


Here we suppose that action-angle variables exist in a suitable region in phase space, whereas $X$ is a globally defined function. Transformation of the Stäckel matrix and potentials

$$
S \rightarrow \tilde{S}=S \cdot \operatorname{diag}\left(k_{1}^{2}, k_{2}^{2}, \ldots, k_{n}^{2}\right), \quad \tilde{V}_{j}=k_{j} V_{j}, \quad k_{i} \in \mathbb{Z},
$$

could preserve superintegrablity, because the same function on new angle variables

$$
\tilde{X}=F\left(\tilde{\omega}_{1}, \tilde{\omega}_{2}, \ldots, \tilde{\omega}_{n}\right)
$$

remains well defined integral of motion. Our main aim is to discuss a few examples of such transformation.

\section{Superintegrable Stäckel systems}

Trajectories of the Stäckel system are defined by equations $I_{j}=\alpha_{j}$ and $\omega_{j}=\beta_{j}(1.2[1.3)$ which form the system of Abel's equations

$$
\frac{1}{n} \sum_{j=1}^{n} \int \frac{S_{i j}\left(u_{j}\right)}{\sqrt{\sum_{m=1}^{n} \alpha_{m} S_{m j}\left(u_{j}\right)-V_{j}\left(u_{j}\right)}} \mathrm{d} u_{j}=\beta_{i}, \quad i=1, \ldots, n .
$$

Solution of this Jacobi inversion problem is simplified if we known algebraic integrals of Abel's equations [1. Various mathematical constructions of these algebraic integrals were discussed by Euler, Jacobi, Abel, Rishelot, Weierstrass, etc. In mechanics algebraic integrals of Abel's equations coincide with the globally defined additional integrals of motion $X_{i}$ (1.1), see discussion in [16, 44, 46, 47.

In 1852 Bertrand proposed a direct method for searching of dynamical systems with bound trajectories on the plane [2] and in 1873 he proved that among central force potentials with bound orbits there are only two types of central force potentials with the property that all bound orbits are also closed orbits 3 . In 1877 a similar theorem about central non-potential forces was established by Darboux and Halphen [8, 19.

In 1870 Korkin applied Bertrand's method to the investigation of dynamical systems with non-central forces [27, which were later generalized by Goryachev, Suslov, Bobylev and Steklov, see textbook [4] and review [39]. In contrast with Bertrand's theorem there are infinitely many systems on the plane with closed trajectories, and now we have a vast literature on superintegrable Stäckel systems which focuses on construction of potentials producing superintegrable systems with periodic dynamics see, e.g., [11, 18, 25, 26, 28, 29, 32, 34, 40.

In [31] Onofri and Pauri came back to Abel's equations (2.4) solving them with respect to Stäckel potentials $V_{j}$. Instead of solving Abel's equations we use known solutions which have been collected in tables of integrals [14].

Let us suppose that angle variables

$$
\omega_{i}=\frac{1}{n} \sum_{j=1}^{n} \int \frac{S_{i j}\left(u_{j}\right)}{\sqrt{\sum_{m=1}^{n} I_{m} S_{m j}\left(u_{j}\right)-V_{j}\left(u_{j}\right)}} \mathrm{d} u_{j}
$$

consist only of integrals of algebraic functions. According to Abel's theorem such integrals and, therefore, angle variables have the following form

$$
\omega_{i}=\sum e_{j}+\sum l_{m}+\sum \rho_{k},
$$

where $e_{j}$ are non-elementary by Liouville functions, $l_{m}$ are logarithmic functions and $\rho_{k}$ are rational functions. It is quite natural to extract the following four families of superintegrable Stäckel systems:

1. $\omega_{i}$ is a sum of rational functions $e_{j}=l_{m}=0$;

2. $\omega_{i}$ is a sum of logarithmic and rational functions $e_{j}=0$; 
3. $\omega_{i}$ is a sum of logarithms $e_{j}=\rho_{k}=0$;

4. $\omega_{i}$ is a sum of non-elementary function $l_{m}=\rho_{k}=0$.

Here and below we impose conditions on each term in the sum $\omega_{i}$ instead of one condition on function $X=F\left(\omega_{i}\right)$ of this sum. We do not consider possible more general cases .

These four families are related to integration of binomials, trinomials and higher order polynomials

$$
\int R\left(x, \sqrt{a+b x^{m}}\right) d x, \quad \int R\left(x, \sqrt{a x^{2}+b x+c}\right) d x, \ldots, \int R\left(x, \sqrt{a_{m} x^{m}+\cdots+a_{0}}\right) d x,
$$

where $R$ is a rational function. All such integrals are well- known, and we can directly take them from the handbook 14 .

Below we present some examples of the known superintegrable Stäckel systems which belong to these four families.

\section{Example 1 - Sum of rational functions:}

Let us start with superintegrable systems separable in Cartesian coordinates $q_{1,2}$ on the plane. The following Stäckel matrix and potentials

$$
S=\left(\begin{array}{cc}
1 & 1 \\
1 & -1
\end{array}\right), \quad V_{1}=a q_{1}^{M_{1}}, \quad V_{2}=b q_{2}^{M_{2}}
$$

give rise to Hamiltonian

$$
H=2 I_{1}=p_{1}^{2}+p_{2}^{2}+V_{1}+V_{2}
$$

which commutes with the second angle variable

$$
\omega_{2}=\int \frac{d q_{1}}{\sqrt{I_{1}+I_{2}-V_{1}\left(q_{1}\right)}}-\int \frac{d q_{2}}{\sqrt{I_{1}-I_{2}-V_{2}\left(q_{2}\right)}}
$$

involving integrals on differential binomials. In 1853 Chebyshev [7] proved that integrals on differential binomials

$$
\int x^{m}\left(\alpha+\beta x^{n}\right)^{p} d x
$$

can be evaluated in terms of elementary functions if and only if:

- $p$ is an integer,

- $\frac{m+1}{n}$ is an integer,

- $\frac{m+1}{n}+p$ is an integer .

For the Stäckel systems

$$
\alpha=I_{1,2}, \quad \beta=1 \quad m=0, \quad n=M, \quad p=-1 / 2
$$

and, therefore, Hamiltonian

$$
H=p_{1}^{2}+p_{2}^{2}+a q_{1}^{M_{1}}+b q_{2}^{M_{2}}, \quad a, b \in \mathbb{R},
$$

is superintegrable, if exponents $M_{1}$ and $M_{2}$ belong to the following sequence of positive rational numbers

$$
M=0,1, \frac{1}{2}, \frac{1}{3}, \frac{1}{4}, \cdots, \frac{1}{n}, \quad n \in \mathbb{Z}_{+},
$$

or sequence of negative rational numbers

$$
M=0,-2,-\frac{2}{3},-\frac{2}{5},-\frac{2}{7}, \cdots,-\frac{2}{2 n-1} .
$$


The corresponding first integrals $X=\left(I_{1}+I_{2}\right)^{m_{1}}\left(I_{1}-I_{2}\right)^{m_{2}} \omega_{2}$ are polynomials with respect to momenta, see details in [17, 25, 18]. For instance, at

$$
H=2 I_{1}=p_{1}^{2}+p_{2}^{2}+a q_{1}^{1 / 5}
$$

second angle variable is the rational function

$$
\omega_{2}=\frac{5 p_{1} q_{1}^{4 / 5}}{a}+\frac{40 p_{1}^{3} q_{1}^{3 / 5}}{3 a^{2}}+\frac{16 p_{1}^{5} q_{1}^{2 / 5}}{a^{3}}+\frac{64 p_{1}^{7} q_{1}^{1 / 5}}{7 a^{4}}+\frac{128 p_{1}^{9}}{63 a^{5}}+\frac{q_{2}}{2 p_{2}},
$$

and additional integral of motion $X=\sqrt{I_{1}-I_{2}} \omega_{2}=p_{2} \omega_{2}$ is polynomial in momenta of tenth order. 39 .

Discussion of similar dynamical systems with non-potential forces may be found in 4, 27,

\section{Example 2 - Sum of rational and logarithmic functions:}

Let us consider superintegrable systems separable in polar coordinates on the plane

$$
r=\sqrt{q_{1}^{2}+q_{2}^{2}}, \quad \phi=\arctan q_{1} / q_{2} .
$$

Standard Stäckel matrix

$$
S=\left(\begin{array}{cc}
1 & 0 \\
r^{-2} & -1
\end{array}\right)
$$

gives rise to the action-angle variables

$$
\begin{aligned}
& I_{1}=p_{r}^{2}+V_{1}(r)+\frac{p_{\phi}^{2}+V_{2}(\phi)}{r^{2}}, \quad \omega_{1}=\frac{1}{2} \int \frac{r d r}{\sqrt{r^{2} I_{1}-r^{2} V_{1}(r)+I_{2}}} \\
& I_{2}=-p_{\phi}^{2}-V_{2}(\phi), \quad \omega_{2}=-\frac{1}{2} \int \frac{d r}{r \sqrt{r^{2} I_{1}-r^{2} V_{1}(r)+I_{2}}}+\frac{1}{2} \int \frac{d \phi}{\sqrt{I_{2}-V_{2}(\phi)}} .
\end{aligned}
$$

At $V_{2}(\phi)=0$ and

$$
V_{1}(r)=a r^{2} \quad \text { or } \quad V_{1}(r)=\frac{a}{r}
$$

second angle variable is the sum of logarithmic and rational functions

$$
\omega_{2}=\frac{\log A_{1}+2 \mathrm{i} \phi}{4 \sqrt{I_{2}}}, \quad A_{1}=\frac{2 I_{2}+r^{2} I_{1}+2 r p_{r} \sqrt{I_{2}}}{r^{2}}
$$

or

$$
\omega_{2}=\frac{\log A_{1}+\mathrm{i} \phi}{2 \sqrt{I_{2}}}, \quad A_{1}=\frac{2 I_{2}-a r+2 r p_{r} \sqrt{I_{2}}}{r} .
$$

More generic potentials were considered in [31].

The following functions on this angle variable are well defined polynomial integrals of motion

$$
X=\exp \left(4 \sqrt{I_{2}} \omega_{2}\right)=A_{1} e^{2 \mathrm{i} \phi}, \quad \text { and } \quad X=\exp \left(2 \sqrt{I_{2}} \omega_{2}\right)=A_{1} e^{\mathrm{i} \phi} .
$$

Taking real or imaginary part of $X$ one obtains real integrals of motion which appear in Bertrand's theorem [2, 3.

For instance, in the second case one gets a Hamiltonian for the superintegrable Kepler system

$$
I_{1}=H=p_{r}^{2}+\frac{a}{r}+\frac{p_{\phi}^{2}}{r^{2}}
$$

with additional integral of motion

$$
X=\exp \left(2 \sqrt{I_{2}} \omega_{2}\right)=\frac{\left(2 \mathrm{i} r p_{r} p_{\phi}-2 p_{\phi}^{2}-a r\right)}{r} e^{\mathrm{i} \phi},
$$


which generates Laplace-Runge-Lenz vector $(\operatorname{Re} X,-\operatorname{Im} X)$ :

$$
\operatorname{Re} X=2\left(p_{1} q_{2}-p_{2} q_{1}\right) p_{2}-\frac{a q_{1}}{\sqrt{q_{1}^{2}+q_{2}^{2}}}, \quad-\operatorname{Im} X=2\left(p_{1} q_{2}-p_{2} q_{1}\right) p_{1}+\frac{a q_{2}}{\sqrt{q_{1}^{2}+q_{2}^{2}}} .
$$

If we take one of the potentials from (2.7) and apply Chebyshev's theorem to the second integral in $\omega_{2}(2.6)$, then one gets an action variable of the form

$$
\omega_{2}=\frac{\log A_{1}+\rho\left(\phi, p_{\phi}\right)}{4 \sqrt{I_{2}}},
$$

where $\rho$ is an algebraic function. The corresponding integral of motion

$$
X=A_{1} \exp \rho\left(\phi, p_{\phi}\right)
$$

is a transcendental function in momenta. Some examples of superintegrable systems with transcendental integrals of motion may be found in [21].

\section{Example 3 - Sum of logarithmic functions:}

Let us continue to consider superintegrable systems separable in polar coordinates on the plane. In order to get a desired sum of the logarithmic function in $\omega_{2}(2.6)$ we make standard trigonometric substitution $u=\cos \phi$ in integral

$$
Z=\frac{1}{2} \int \frac{d \phi}{\sqrt{I_{2}-V_{2}(\phi)}}=-\frac{1}{2} \int \frac{d u}{\sqrt{\left(1-u^{2}\right)\left(I_{2}-V_{2}(u)\right.}}
$$

and look over potentials $V_{2}(u)$ associated with Euler's substitutions

$$
\begin{aligned}
V_{2}(u) & =\frac{\alpha u+\beta}{u^{2}-1}, & Z & =-\frac{1}{2} \int \frac{d u}{\sqrt{-I_{2} u^{2}+\alpha u+\left(I_{2}+\beta\right)}} \\
V_{2}(u) & =\frac{\alpha u^{2}+\beta}{u^{2}\left(u^{2}-1\right)}, & Z & =-\frac{1}{2} \int \frac{u d u}{\sqrt{-I_{2} u^{4}+\left(I_{2}+\alpha\right) u^{2}+\beta}} .
\end{aligned}
$$

These integrals are equal to

$$
\begin{array}{ll}
Z=\frac{1}{2 \sqrt{I_{2}}} \log A_{2}, & A_{2}=2 I_{2} \cos \phi+\alpha+2 \sqrt{I_{2}} p_{\phi} \sin \phi, \\
Z=\frac{\mathrm{i}}{4 \sqrt{I_{2}}} \log A_{2}, & A_{2}=I_{2} \cos 2 \phi+\alpha+\sqrt{I_{2}} p_{\phi} \sin 2 \phi .
\end{array}
$$

As a result, we obtain angle variable

$$
\omega_{2}=\frac{1}{4 \sqrt{I_{2}}}\left(m_{1} \log A_{1}+m_{2} \log A_{2}\right)=\frac{1}{\sqrt{I_{2}}} \log \left(A_{1}^{m_{1}} A_{2}^{m_{2}}\right)
$$

and recover the well-known additional integral of motion

$$
X=\exp 4 \sqrt{I_{2}} \omega_{2}=A_{1}^{m_{1}} A_{2}^{m_{2}}, \quad m_{k}=1,2 .
$$

for superintegrable system with Hamiltonian

$$
H=I_{1}=p_{r}^{2}+V_{1}(r)+\frac{p_{\phi}^{2}+V_{2}(\phi)}{r^{2}}
$$

where $V_{1}(r)$ is equal to (2.7) and

$$
V_{2}(\phi)=\frac{\alpha \cos \phi+\beta}{\cos ^{2} \phi-1} \quad \text { and } \quad V_{2}(\phi)=\frac{\alpha \cos ^{2} \phi+\beta}{\cos ^{2} \phi\left(\cos ^{2} \phi-1\right)} .
$$


For instance, taking the first potentials from (2.7) and second from (2.10) we obtain the wellknown Smorodinsky-Winternitz superintegrable system [10] with Hamiltonian

$$
\begin{aligned}
H & =p_{1}^{2}+p_{2}^{2}+a\left(q_{1}^{2}+q_{2}^{2}\right)+\frac{b_{1}}{q_{1}^{2}}+\frac{b_{2}}{q_{2}^{2}} \\
& =p_{r}^{2}+\frac{p_{\phi}^{2}}{r^{2}}+a r^{2}+\frac{b_{1}}{r^{2} \sin ^{2} \phi}+\frac{b_{2}}{r^{2} \cos ^{2} \phi},
\end{aligned}
$$

where $b_{1}=-\alpha-\beta$ and $b_{2}=-\beta$.

Using another trigonometric substitution $u=\cos k \phi$ one gets superintegrable systems with additional integrals of motion which are higher order polynomials in momenta. We discuss these systems in the next Section.

\section{Example 4 - Sum of elliptic integrals:}

Let us consider superintegrable system separable in elliptic coordinates on the plane $u_{1,2}$ defined by

$$
1-\frac{q_{1}^{2}}{\lambda-\kappa}-\frac{q_{2}^{2}}{\lambda+\kappa}=\frac{\left(\lambda-u_{1}\right)\left(\lambda-u_{2}\right)}{\lambda^{2}-\kappa^{2}}, \quad \kappa \in \mathbb{R} .
$$

The corresponding Stäckel matrix

$$
S=\left(\begin{array}{cc}
\frac{u_{1}}{u_{1}^{2}-\kappa^{2}} & \frac{u_{2}}{u_{2}^{2}-\kappa^{2}} \\
\frac{1}{u_{1}^{2}-\kappa^{2}} & \frac{1}{u_{2}^{2}-\kappa^{2}}
\end{array}\right),
$$

determines action variable

$$
I_{1}=\frac{\left(u_{1}^{2}-\kappa^{2}\right)\left(p_{u_{1}}^{2}+V_{1}\right)}{u_{1}-u_{2}}+\frac{\left(u_{2}^{2}-\kappa^{2}\right)\left(p_{u_{2}}^{2}+V_{2}\right)}{u_{2}-u_{1}}
$$

and angle variable

$$
\omega_{2}=e_{1}+e_{2}, \quad e_{i}=\frac{1}{2} \int \frac{d u_{i}}{\sqrt{\left(u_{i}^{2}-\kappa^{2}\right)\left(u_{i} I_{1}+I_{2}-V_{i}\left(u_{i}\right)\left(u_{i}^{2}-\kappa^{2}\right)\right)}},
$$

which is a sum of elliptic integrals at

$$
V_{j}(u)=4\left(a+\frac{b_{1}}{(\kappa-u)^{2}}+\frac{b_{2}}{(\kappa+u)^{2}}\right)
$$

Using standard divisor arithmetics on the corresponding elliptic curve, we can easily obtain an additional integral of motion for the Smorodinsky-Winternitz superintegrable system [10]

$$
\begin{aligned}
H & =p_{1}^{2}+p_{2}^{2}+a\left(q_{1}^{2}+q_{2}^{2}\right)+\frac{b_{1}}{q_{1}^{2}}+\frac{b_{2}}{q_{2}^{2}} \\
& =\frac{\left(u_{1}^{2}-\kappa^{2}\right) p_{u_{1}}^{2}-\left(u_{2}-\kappa^{2}\right) p_{u_{2}}^{2}}{4\left(u_{1}-u_{2}\right)}+a\left(u_{1}+u_{2}\right)-\frac{2 \kappa b_{1}}{\left(\kappa-u_{1}\right)\left(\kappa-u_{2}\right)}+\frac{2 \kappa b_{2}}{\left(\kappa+u_{1}\right)\left(\kappa+u_{2}\right)},
\end{aligned}
$$

which coincides with Euler algebraic integral for Abel's equations, see details in [46, 48].

In the next Section we discuss transformation of the Stäckel matrix which allows us to get an infinite family of superintegrable systems starting with the known superintegrable Stäckel system. 


\section{Transformation of the Stäckel matrix}

Our main idea is to take a superintegrable Stäckel system and make some variables "faster" or "slower". Indeed, multiplying columns of the Stäckel matrix and potentials on integers

$$
\tilde{S_{i j}}=k_{j}^{2} S_{i j}, \quad \tilde{V}_{j}=k_{j} V_{j}, \quad k_{j} \in \mathbb{Z},
$$

we change separated relations

$$
\begin{aligned}
p_{u_{j}}^{2} & =\sum I_{i} S_{i j}\left(u_{j}\right)-V_{j}\left(u_{j}\right), \\
k_{j}^{-2} p_{u_{j}}^{2} & =\sum I_{i} S_{i j}\left(u_{j}\right)-V_{j}\left(u_{j}\right),
\end{aligned}
$$

and angle variables

$$
\begin{aligned}
& \omega_{j}=\frac{1}{n}\left(\int \frac{d u_{1}}{p_{u_{1}}}+\int \frac{d u_{2}}{p_{u_{2}}}+\cdots+\int \frac{d u_{n}}{p_{u_{n}}}\right) \\
& \tilde{\omega}_{j}=\frac{1}{n}\left(k_{1} \int \frac{d u_{1}}{p_{u_{1}}}+k_{2} \int \frac{d u_{2}}{p_{u_{2}}}+\cdots+k_{n} \int \frac{d u_{n}}{p_{u_{n}}}\right) .
\end{aligned}
$$

In fact, this transformation of the Stäckel matrix and potentials is equivalent to non-canonical transformation of momenta $p_{u_{j}} \rightarrow k_{j}^{-1} p_{u_{j}}$.

If angle variable $\omega_{j}$ is a sum of rational function, then variable $\tilde{\omega}_{j}$ is also a sum of rational function

$$
\begin{aligned}
& \omega_{j}=\rho_{1}+\rho_{2}+\cdots+\rho_{n}=R_{j} \\
& \tilde{\omega}_{j}=k_{1} \rho_{1}+k_{2} \rho_{2}+\cdots+k_{n} \rho_{n}=\tilde{R}_{j},
\end{aligned}
$$

which can be also used for construction of additional integrals of motion which are polynomials in momenta.

If $\omega_{j}$ is a sum of logarithmic functions, then variable $\tilde{\omega}_{j}$ is also a sum of logarithms

$$
\begin{aligned}
& \omega_{j}=g(I)\left(\ln A_{1}+\ln A_{2}+\cdots+\ln A_{n}\right)=g(I) \ln \left(A_{1} A_{2} \cdots A_{n}\right) \\
& \tilde{\omega}_{j}=g(I)\left(k_{1} \ln A_{1}+k_{2} \ln A_{2}+\cdots+k_{n} \ln A_{n}\right)=g(I) \ln \left(A_{1}^{k_{1}} A_{2}^{k_{2}} \cdots A_{n}^{k_{n}}\right),
\end{aligned}
$$

where $g(I)$ is a function on action variables as in (2.9). In this case transformation of the Stäckel matrix changes the original additional integral of motion

$$
X=A_{1} A_{2} \cdots A_{n} \rightarrow \tilde{X}=A_{1}^{k_{1}} A_{2}^{k_{2}} \cdots A_{n}^{k_{n}},
$$

that allows us to get superintegrable systems with additional integrals of motion which are polynomials of higher order in momenta.

If $\omega_{j}$ is a sum of elliptic (hyperelliptic) integrals as in (2.13), then divisors arithmetics can also be applied to $\tilde{\omega}_{j}$

$$
\omega_{j}=e_{1}+\cdots+e_{n}, \quad \tilde{\omega}_{j}=k_{1} e_{1}+\cdots+k_{n} e_{n},
$$

see discussion of addition and multiplication of elliptic integrals in 20. In this case well-defined additional integrals of motion $X$ could be rational functions in momenta. We plan to discuss the relations of superintegrable systems and arithmetic of divisors in further publications.

Thus, we suppose that the following Proposition can be true.

Proposition 1 Transformation of the Stäckel matrix and potentials

$$
S \rightarrow \tilde{S}=S \cdot \operatorname{diag}\left(k_{1}^{2}, k_{2}^{2}, \ldots, k_{n}^{2}\right), \quad V_{j} \rightarrow \tilde{V}_{j}=k_{j} V_{j}, \quad k_{j} \in \mathbb{Z}
$$

preserves superintegrability. 
We can prove this Proposition only in partial cases at $n=2,3$.

Transformation (3.16) changes diagonal metric $g_{j j} \rightarrow \tilde{g}_{j j}=g_{j j} k_{i}^{-2}$ in the original Hamiltonian

$$
H \rightarrow \tilde{H}=\tilde{I}_{1}=\sum \tilde{\mathrm{g}}_{j j} p_{u_{j}}^{2}+V\left(u_{1}, \ldots, u_{n}\right)=\sum \mathrm{g}_{j j}\left(\frac{p_{u_{j}}}{k_{j}}\right)^{2}+V\left(u_{1}, \ldots, u_{n}\right) .
$$

After this transformation the new metric could be

- equivalent to the original metric;

- non-equivalent to the original metric.

In [26] one can find examples of such transformations in $\mathbb{R}^{3}$, which transform flat metric to the non-flat metric and vise versa. Here we consider only some examples of superintegrable Stäckel systems with two degrees of freedom.

\section{Example 5 - System separable in Cartesian coordinates}

In this case transformation of the Stäckel matrix (3.16)

$$
S=\left(\begin{array}{cc}
1 & 1 \\
1 & -1
\end{array}\right) \rightarrow \tilde{S}=\left(\begin{array}{cc}
k_{1}^{2} & k_{2}^{2} \\
k_{1}^{2} & -k_{2}^{2}
\end{array}\right)
$$

does not change properties of the original metric

$$
\mathrm{g}=\left(\begin{array}{ll}
1 & 0 \\
0 & 1
\end{array}\right) \rightarrow \tilde{\mathrm{g}}=\left(\begin{array}{cc}
k_{1}^{-2} & 0 \\
0 & k_{2}^{-2}
\end{array}\right)
$$

due to the existence of additional canonical transformation $p_{i} \rightarrow k_{i} p_{i}$ and $q_{i} \rightarrow k_{i}^{-1} q_{i}$, which allows us to change the potential instead of metric.

For instance, Hamiltonian of the Smorodinsky-Winternitz superintegrable system

$$
H=p_{1}^{2}+p_{2}^{2}+a\left(q_{1}^{2}+q_{2}^{2}\right)+\frac{b_{1}}{q_{1}^{2}}+\frac{b_{2}}{q_{2}^{2}}
$$

after transformation (3.16) looks like

$$
\tilde{H}=\left(\frac{p_{1}}{k_{1}}\right)^{2}+\left(\frac{p_{2}}{k_{2}}\right)^{2}+a\left(q_{1}^{2}+q_{2}^{2}\right)+\frac{b_{1}}{q_{1}^{2}}+\frac{b_{2}}{q_{2}^{2}} .
$$

After canonical transformation $p_{i} \rightarrow k_{i} p_{i}$ and $q_{i} \rightarrow k_{i}^{-1} q_{i}$ one gets Hamiltonian

$$
\tilde{H}=p_{1}^{2}+p_{2}^{2}+a k_{1}^{-2} q_{1}^{2}+a k_{2}^{-2} q_{2}^{2}+\frac{k_{1}^{2} b_{1}}{q_{1}^{2}}+\frac{k_{2}^{2} b_{2}}{q_{2}^{2}}
$$

of the well-known superintegrable system with integral of motion which is higher order polynomial in momenta associated with logarithmic angle variable $\tilde{\omega}_{2}$ of the form (3.15) [17, 29].

Example 6 - System separable in polar coordinates

In this case transformation of the Stäckel matrix (3.16)

$$
S=\left(\begin{array}{cc}
1 & 0 \\
r^{-2} & -1
\end{array}\right) \rightarrow \tilde{S}=\left(\begin{array}{cc}
k_{1}^{2} & 0 \\
k_{1}^{2} r^{-2} & -k_{2}^{2}
\end{array}\right)
$$

also does not change properties of the original metric

$$
\mathrm{g}=\left(\begin{array}{cc}
1 & 0 \\
0 & r^{-2}
\end{array}\right) \rightarrow \tilde{g}=\left(\begin{array}{cc}
k_{1}^{-2} & 0 \\
0 & k_{2}^{-2} r^{-2}
\end{array}\right)
$$


For instance, let us consider superintegrable Hamiltonians

$$
H=I_{1}=p_{r}^{2}+\frac{p_{\phi}^{2}}{r^{2}}+V_{1}(r)+\frac{V_{2}(\phi)}{r^{2}}
$$

where Stäckel potentials $V_{1,2}$ are given by (2.7) and (2.10). After transformation (3.16) we obtain the following Hamiltonian

$$
\tilde{H}=\left(\frac{p_{r}}{k_{1}}\right)^{2}+\frac{1}{r^{2}}\left(\frac{p_{\phi}}{k_{2}}\right)^{2}+V_{1}(r)+\frac{V_{2}(\phi)}{r^{2}} .
$$

Canonical transformation $r \rightarrow k_{1}^{-1} r$ and $\phi \rightarrow k_{2}^{-1} \phi$ reduces metric to the original one and changes potential

$$
\tilde{H}=p_{r}^{2}+\frac{p_{\phi}^{2}}{r^{2}}+V_{1}\left(k_{1}^{-1} r\right)+\frac{V_{2}\left(k_{2}^{-1} \phi\right)}{k_{1}^{-2} r^{2}} .
$$

As a result, one gets the well-known superintegrable systems with additional integrals of motion $X$, which are polynomials in momenta of higher order associated with logarithmic angle variable $\tilde{\omega}_{2}$ of the form (3.15), see [12, 26, 32, 34, 41].

For instance, we can reconstruct a second family of superintegrable Hamiltonians associated with Smorodinsky-Winternitz superintegrable system (2.11)

$$
\tilde{H}=p_{r}^{2}+\frac{p_{\phi}^{2}}{r^{2}}+a r^{2}+\frac{b_{1}}{r^{2} \sin ^{2} k \phi}+\frac{b_{2}}{r^{2} \cos ^{2} k \phi}
$$

which can be also obtained using trigonometric substitution $u=\cos k \phi$ in (2.8).

\section{Example 7 - Systems separable in parabolic coordinates}

Let us consider superintegrable systems separable in parabolic coordinates $u_{1,2}$ on the plane defined by

$$
q_{1}=u_{1} u_{2}, \quad q_{2}=\left(u_{1}^{2}-u_{2}^{2}\right) / 2 .
$$

The corresponding Stäckel matrix

$$
S=\left(\begin{array}{cc}
u_{1}^{2} & u_{2}^{2} \\
1 & -1
\end{array}\right)
$$

determines action variables

$$
I_{1}=\frac{p_{u_{1}}^{2}+V_{1}\left(u_{1}\right)+p_{u_{2}}^{2}+V_{2}\left(u_{2}\right)}{u_{1}^{2}+u_{2}^{2}}, \quad I_{2}=\frac{u_{2}^{2}\left(p_{u_{1}}^{2}+V_{1}\left(u_{1}\right)\right)-u_{1}^{2}\left(p_{u_{2}}^{2}+V_{2}\left(u_{2}\right)\right)}{u_{1}^{2}+u_{2}^{2}}
$$

and angle variables

$$
\begin{aligned}
& \omega_{1}=-\frac{1}{2}\left(\int \frac{u_{1}^{2} d u_{1}}{\sqrt{u_{1}^{2} I_{1}-V_{1}\left(u_{1}\right)+I_{2}}}+\int \frac{u_{2}^{2} d u_{2}}{\sqrt{u_{2}^{2} I_{1}-V_{2}\left(u_{2}\right)-I_{2}}}\right) \\
& \omega_{2}=-\frac{1}{2}\left(\int \frac{d u_{1}}{\sqrt{u_{1}^{2} I_{1}-V_{1}\left(u_{1}\right)+I_{2}}}-\int \frac{d u_{2}}{\sqrt{u_{2}^{2} I_{1}-V_{2}\left(u_{1}\right)-I_{2}}}\right) .
\end{aligned}
$$

In order to get a desired sum of logarithms we reduce integrals to the following form

$$
\int R\left(x, \sqrt{a x^{2}+b x+c}\right) d x,
$$

where $R$ is a rational function, and apply the so-called Euler's substitutions. One gets logarithms for the following potentials only:

$$
V(u)=a u^{2}+b u+c \quad \Rightarrow \quad \int \frac{d u}{\sqrt{\left(I_{1}-a\right) u^{2}-b u+I_{2}-c}}
$$


and

$$
V^{\prime}(u)=\frac{a u^{4}+b u^{2}+c}{u^{2}} \Rightarrow \int \frac{u d u}{\sqrt{\left(I_{1}-a\right) u^{4}+\left(I_{2}-b\right) u^{2}-c}} .
$$

The corresponding Hamiltonian commutes with second angle variable

$$
\omega_{2}=\frac{1}{2 \sqrt{I_{1}-a_{1}}} \log A_{1}+\frac{1}{2 \sqrt{I_{1}-a_{2}}} \log A_{2},
$$

which is the sum of two logarithms with a common factor at $a_{1}=a_{2}=$ const only. This constant is irrelevant since it can always be associated with a shift of $I_{1}$, so we put $a_{1}=a_{2}=0$. As a result we have three superintegrable Hamiltonains

$$
H^{(j)}=I_{1}=\frac{p_{u_{1}}^{2}+p_{u_{2}}^{2}}{u_{1}^{2}+u_{2}^{2}}+U_{j}\left(u_{1}, u_{2}\right), \quad j=1,2,3
$$

where

$$
U_{1}=\frac{V\left(u_{1}\right)+V\left(u_{2}\right)}{u_{1}^{2}+u_{2}^{2}}, \quad U_{2}=\frac{V\left(u_{1}\right)+V^{\prime}\left(u_{2}\right)}{u_{1}^{2}+u_{2}^{2}}, \quad U_{3}=\frac{V^{\prime}\left(u_{1}\right)+V^{\prime}\left(u_{2}\right)}{u_{1}^{2}+u_{2}^{2}} .
$$

Additional integrals of motion are equal to

$$
X_{1}=\exp \left(2 \sqrt{I_{1}} \omega_{2}\right)=A_{1} A_{2}, \quad X_{2}=A_{1} A_{2}^{\prime}, \quad X_{3}=A_{1}^{\prime} A_{2}^{\prime},
$$

where

$$
A_{j}=\frac{2 I_{1} u_{j}+2 \mathrm{i} p_{u_{j}} \sqrt{I_{1}}-b_{j}}{2 \sqrt{I_{1}}} \quad \text { and } \quad A_{j}^{\prime}=\left(\frac{2 I_{1} u_{j}^{2}+I_{2}+2 \mathrm{i} u_{j} p_{u_{j}} \sqrt{I_{1}}-b_{j}}{2 \sqrt{I_{1}}}\right)^{2} .
$$

Taking real or imaginary part of $X_{k}$ one obtains real integrals of motion functionally independent on action variables $I_{1,2}$. Obviously, in this way we produce only one new independent integral for each Hamiltonian.

Transformation of the Stäckel matrix

$$
S=\left(\begin{array}{cc}
u_{1}^{2} & u_{2}^{2} \\
1 & -1
\end{array}\right) \rightarrow \tilde{S}=\left(\begin{array}{cc}
k_{1}^{2} u_{1}^{2} & k_{2}^{2} u_{2}^{2} \\
k_{1}^{2} & -k_{2}^{2}
\end{array}\right)
$$

changes metric in Hamiltonians 3.19)

$$
\mathrm{g}=\frac{1}{u_{1}^{2}+u_{2}^{2}}\left(\begin{array}{ll}
1 & 0 \\
0 & 1
\end{array}\right) \rightarrow \tilde{\mathrm{g}}=\frac{1}{u_{1}^{2}+u_{2}^{2}}\left(\begin{array}{cc}
k_{1}^{-2} & 0 \\
0 & k_{2}^{-2}
\end{array}\right)
$$

second angle variable $(3.15)$ and additional integrals of motion

$$
\tilde{X}_{1}=A_{1}^{k_{1}} A_{2}^{k_{2}}, \quad \tilde{X}_{2}=A_{1}^{k_{1}} A_{2}^{\prime k_{2}} \quad \tilde{X}_{3}=A_{1}^{\prime k_{1}} A_{2}^{\prime k_{2}} .
$$

Taking real or imaginary part of $\tilde{X}_{k}$ we obtain additional integrals of motion which are polynomials in momenta of order $m=3,4, \ldots$. Such superintegrable systems have not been researched in the literature before.

\section{Example 8 - Systems separable in elliptic coordinates}

Let us continue to study Hamilton-Jacobi equation $H=E$ for the Smorodinsky-Winternitz superintegrable system which admits separation of variables in Cartesian, polar and elliptic coordinates on the plane.

In elliptic coordinates Hamiltonian (2.14) is equal to

$$
H=\frac{\left(u_{1}^{2}-\kappa^{2}\right) p_{u_{1}}^{2}-\left(u_{2}-\kappa^{2}\right) p_{u_{2}}^{2}}{4\left(u_{1}-u_{2}\right)}+a\left(u_{1}+u_{2}\right)-\frac{2 \kappa b_{1}}{\left(\kappa-u_{1}\right)\left(\kappa-u_{2}\right)}+\frac{2 \kappa b_{2}}{\left(\kappa+u_{1}\right)\left(\kappa+u_{2}\right)} .
$$

Using transformation (3.16) one gets new Hamiltonians

$$
\begin{aligned}
\tilde{H} & =\frac{u_{1}^{2}-\kappa^{2}}{4\left(u_{1}-u_{2}\right)}\left(\frac{p_{u_{1}}}{k_{1}}\right)^{2}-\frac{u_{2}-\kappa^{2}}{4\left(u_{1}-u_{2}\right)}\left(\frac{p_{u_{2}}}{k_{2}}\right)^{2} \\
& +a\left(u_{1}+u_{2}\right)-\frac{2 \kappa b_{1}}{\left(\kappa-u_{1}\right)\left(\kappa-u_{2}\right)}+\frac{2 \kappa b_{2}}{\left(\kappa+u_{1}\right)\left(\kappa+u_{2}\right)}
\end{aligned}
$$


commuting with a second action variable $I_{2}$. At $k_{1}=1,2,3$ and $k_{2}$ we can also get additional integral of motion $X$ associated with angle variable $\omega_{2}(2.13)$ using explicit formulae for doubling and tripling divisors on elliptic curves from [20. In contrast with (3.17) and (3.18) additional integral $X$ is the globally defined rational function on the projective plane.

Summing up, we suppose that the well-known Smorodinsky-Winternitz superintegrable system belongs to the three infinite families of superintegrable systems (3.17), (3.18) and (3.20).

\section{Example 9 - System with masses depending on coordinates}

Let us consider superintegrable Hamiltonian

$$
H=I_{1}=\left(q_{1}^{2}+q_{2}^{2}\right)^{3}\left(p_{1}^{2}+p_{2}^{2}\right)+a q_{1}
$$

with masses depending on coordinates [40]. Substituting this Hamiltonian into the computer program [15] one gets variables of separation

$$
u_{1}=\frac{q_{1}^{2}}{\left(q_{1}^{2}+q_{2}^{2}\right)^{2}}, \quad u_{2}=-\frac{q_{2}^{2}}{\left(q_{1}^{2}+q_{2}^{2}\right)^{2}}
$$

characteristic Killing tensor generating second quadratic integral of motion, Stäckel matrix

$$
S=\left(\begin{array}{cc}
\frac{1}{4} & \frac{1}{4} \\
\frac{1}{u_{1}} & \frac{1}{u_{2}}
\end{array}\right)
$$

second angle variable

$$
\omega_{2}=-\frac{1}{\sqrt{I_{1}}}\left(2 \log A_{1}+\log A_{2}\right)
$$

commuting with $H=I_{1}$ and the globally defined additional integral of motion

$$
X=\exp \left(-\sqrt{I_{1}} \omega_{2}\right)=A_{1}^{2} A_{2},
$$

where

$$
A_{1}=\frac{I_{1} \sqrt{u_{1}}+2 \mathrm{i} p_{u_{1}} \sqrt{u_{1}} \sqrt{I_{1}}-a / 2}{\sqrt{I_{1}}}, \quad A_{2}=\frac{I_{1} u_{2}+2 I_{2}+2 \mathrm{i} p_{u_{2}} u_{2} \sqrt{I_{1}}}{\sqrt{I_{1}}} .
$$

After transformation of the Stäckel matrix (3.16)

$$
S=\left(\begin{array}{cc}
\frac{1}{4} & \frac{1}{4} \\
\frac{1}{u_{1}} & \frac{1}{u_{2}}
\end{array}\right) \rightarrow \tilde{S}=\left(\begin{array}{cc}
\frac{k_{1}^{2}}{4} & \frac{k_{2}^{2}}{4} \\
\frac{k_{1}^{2}}{u_{1}} & \frac{k_{2}^{2}}{u_{2}}
\end{array}\right)
$$

one gets superintegrable Hamiltonian

$$
\tilde{H}=\tilde{I}_{1}=\frac{4 u_{1}}{u_{1}-u_{2}}\left(\frac{p_{u_{1}}}{k_{1}}\right)^{2}+\frac{4 u_{2}}{u_{2}-u_{1}}\left(\frac{p_{u_{2}}}{k_{2}}\right)^{2}+\frac{a \sqrt{u_{1}}}{u_{1}-u_{2}}
$$

with additional integral of motion

$$
\tilde{X}=\exp \left(-\sqrt{\tilde{I}_{1}} \tilde{\omega}_{2}\right)=A_{1}^{2 k_{1}} A_{2}^{k_{2}}
$$

which is independent from action variables. Taking real or imaginary part of $\tilde{I}^{2 k_{1}+k_{2}} \tilde{X}$ we obtain two real integrals of motion, which are polynomials in momenta.

In similar manner we can get infinite families of superintegrable systems associated with superintegrable systems with masses depending on coordinates from [11, 35, 40] 


\section{Conclusion}

For the given Stäckel matrix $S$ we can calculate all the potentials $V_{j}$ for which angle variables (1.2) consist of rational, logarithmic and non-elementary functions. Then we can apply transformation of the Stäckel matrix and potentials (3.16) to construct infinite families of superintegrable systems. For instance, we can start with Stäckel matrices $S$ associated with orthogonal curvilinear coordinate systems in spaces of constant curvature from [30. For instance, there are 4 coordinate systems and Stäckel matrices for $\mathbb{E}^{2} ; 2$ systems for $\mathbb{S}^{2} ; 9$ systems for $\mathbb{H}^{2}$ and 11 systems for $\mathbb{E}^{3} ; 6$ systems for $\mathbb{S}^{3} ; 45$ system in $\mathbb{H}^{3}$. In this paper we present some examples of such construction including Smorodinsky-Winternitz system, which generates three infinite families of superintegrable systems.

More complicated construction of superintegrable systems involves non-point canonical transformation relating original variables and variables of separation. If matrix $S$ and potentials $V_{j}$ depend on parameter $a$, then action-angle variables (1.2]1.3) also depend on this parameter. After non-point canonical transformation

$$
q_{i}=f_{i}\left(u, p_{u}, a\right), \quad p_{i}=g_{i}\left(u, p_{u}, a\right)
$$

angle variables $\omega_{i}(q, p, a)$ could be used to construct an additional integral of motion $X=F\left(\omega_{i}\right)$ which becomes a globally defined function only at $a \rightarrow 0$. Examples of such superintegrable systems may be found in [6, 33, 49]. It will be interesting to apply transformation of the Stäckel matrix and potentials (3.16) to these systems.

The work was supported by the RFBR grant (project 18-01-00916).

\section{References}

[1] Baker H. F., Abel's theorem and the allied theory of theta functions, Cambridge Univ. Press, Cambridge, 1897.

[2] Bertrand J., Mémoire sur les intégrales communes à plusieurs problémes se Mécanique, Journal Mathématiques pures et appliquées, ser 1, v.XVII, pp. 121-174, (1852).

[3] Bertrand J., Théorème relatif au mouvement dun point attiré vers un centre fixe, Comptes rendus de l'Acadmie des sciences de Paris, v.77, pp. 849-853, (1873).

[4] Bobylev D.K., Course of analytical mechanics, v.2, Petersburg, (1883).

[5] Boyer C.P., Kalnins E.G., Miller Jr. W., Stäckel-equivalent integrable Hamiltonian systems, SIAM J. Math. Anal., v. 17), pp.778-797, (1986).

[6] Campoamor-Stursberg R., Cariñena J. F., Rañada M.F., Higher-order superintegrability of a Holt related potential, J. Phys. A: Math. Theor., v.46(43), 435202, 2013.

[7] Chebyshev P.L., Sur l'intégration des différentielles irrationnelles, J. Math. Pures Appl., v.18, pp. 87-111, (1853).

[8] Darboux M.G. Recherche de la loi que doit suivre une force centrale, pour que la trajectoire quelle détermine soit toujours une conique, Comptes rendus de lAcadmie des sciences de Paris, v.84, pp.760-936, 1877.

[9] Fassò F., Superintegrable Hamiltonian Systems: Geometry and Perturbations, Acta Applicandae Mathematicae, v. 87, pp. 93121, (2005).

[10] Fris̃ T.I., Mandrosov V., Smorodinsky Y.A., Uhlir̃ M., Winternitz P., On higher symmetries in quantum mechanics, Phys. Lett., v.16(3), pp.354-356, (1965).

[11] Ghose-Choudhury A., Guha P., Superintegrable systems with position dependent mass: master symmetry and action-angle methods, arXiv preprint arXiv:1804.01074, (2018). 
[12] Gonera C., On the superintegrability of TTW model, Physics Letters A, v.376, pp.2341$2343,(2012)$.

[13] Gonera C., Kaszubska M., Superintegrable systems on spaces of constant curvature, Annals of Physics, v.346, pp.91-102, (2014).

[14] Gradshtein I. S., Ryzhik I. M., Tables Of Integrals, Series And Products, 7th Edition, Academic Press, (2007).

[15] Grigoriev Yu.A., Tsiganov A.V., Symbolic software for separation of variables in the Hamilton-Jacobi equation for the L-systems, Regular and Chaotic Dynamics,, v.10(4), p.413-422, (2005).

[16] Grigoryev Yu. A., Khudobakhshov V. A., Tsiganov A.V., On the Euler superintegrable systems, J. Phys. A: Math. Theor., v.42, 075202, (11pp), (2009).

[17] Grigoriev Yu.A., Tsiganov A.V., On superintegrable systems separable in Cartesian coordinates, Phys. Lett. A, v. 382(32), pp.2092-2096, (2018).

[18] Güngör, Kuru S., Negro J., Nieto L.M., Heisenberg-type higher order symmetries of superintegrable systems separable in cartesian coordinates, Nonlinearity, v.30, pp.17881808, (2017).

[19] Halphen M., Sur les lois de Kepler. Solution d'un probléme proposé par M. Bertrand Comptes rendus de l'Académie des sciences de Paris, v.84, pp.939-942, (1877).

[20] Handbook of Elliptic and Hyperelliptic Curve Cryptography, ed. H. Cohen and G. Frey, Chapman and Hall/CRC, (2006).

[21] Hietarinta J., New integrable Hamiltonians with transcendental invariants, Phys. Rev. Lett., v.52(13), pp.1057-1060, (1984).

[22] Hietarinta J., Grammaticos B., Dorizzi B., Ramani A., Coupling-constant metamorphosis and duality between integrable Hamiltonian systems, Phys. Rev. Lett., v.53(18), pp.17071710, (1984).

[23] Jacobi C. G. J., Ueber die Reduction der Integration der partiellen Differentialgleichungen erster Ordnung zwischen irgend einer Zahl Variablen auf die Integration eines einzigen Systemes gewöhnlicher Differentialgleichungen, Crelle Journal für die reine und angewandte Mathematik, v.17, pp. 97-162, (1837).

[24] Jacobi C. G. J., Note sur l'intégration des équations différentielles de la Dynamique, Comptes rendus de l'Acadmie des sciences de Paris, v. 5, pp. 61-67, (1837).

[25] Kalnins E. G., Kress J. M., Miller W., Jr., Pogosyan G. S., Complete sets of invariants for dynamical systems that admit a separation of variables, J. Math. Phys., v.43(7), pp.35923609, (2002).

[26] Kalnins E. G., Kress J. M., Miller W., Jr., Families of classical subgroup separable superintegrable systems, J. Phys. A: Math. and Theor., v.43(9), 092001, (2010).

[27] Korkine A.N., Sur les intégrales des équations du mouvement d'un point matériel, Math. Ann. v.2, pp. 13-40, (1870).

[28] Maciejewski A.J., Przybylska M., Tsiganov A.V., On algebraic construction of certain integrable and super-integrable systems, Physica D, v. 240, p.1426-1448, (2011).

[29] Miller Jr. W., Post S., Winternitz P., Classical and quantum superintegrability with applications, J. Phys. A: Math. Theor., v.46, 423001, (2013). 
[30] Olevskii M.N., Triorthogonal systems in spaces of constant curvature in which the equation $\Delta_{2} u+\lambda u=0$ allows a complete separation of variables, Mat. Sbornik., v.27(69), pp. 379426, 1950.

[31] Onofri, E. and Pauri, M., Search for periodic hamiltonian flows: A generalized Bertrands theorem, J. Math. Phys., v. 19( 9), pp. 1850-1858, (1978).

[32] Post S., Winternitz P., An infinite family of superintegrable deformations of the Coulomb potential, J. Phys. A: Math. Theor., v. 42, 222001, (2010).

[33] Post S., Winternitz P., A nonseparable quantum superintegrable system in $2 D$ real Euclidean space, J. Phys. A: Math. Theor., v.44(16), 162001, (2011).

[34] Rañada M. F., Higher order superintegrability of separable potentials with a new approach to the Post-Winternitz system, J. Phys. A: Math. Theor., v.46, 125206, (2013).

[35] Rañada M. F., Superintegrable systems with a position dependent mass: Kepler-related and oscillator-related systems, Phys. Lett. A, v. 380 (27-28), pp.2204-2210, (2016).

[36] Reshetikhin N., Degenerately integrable systems, J. Math. Sciences, v.213(5), pp. 769-785, (2016).

[37] Sergyeyev A., Błaszak M., Generalized Stäckel transform and reciprocal transformations for finite-dimensional integrable systems, J. Phys. A: Math. Theor., v.41(10), 105205, 2008.

[38] Stäckel P., Über die Integration der Hamilton-Jacobischen Differential Gleichung Mittelst Separation der Variabeln, Habilitationsschrift, Halle, 26pp., (1891).

[39] Steklov V.A. On a transformation of the differential equations of motion of a free material point in the plane and its applications, Trudy Otdeleniya fizicheskikh nauk Obshchestva lyubiteley estestvoznaniya, v.9, n.1, pp.16-26, (1897).

[40] Szumiński W., Maciejewski A.J., Przybylska M., Note on integrability of certain homogeneous Hamiltonian systems, Phys. Lett. A, v. 379(45-46), pp. 2970-2976, (2015).

[41] Tremblay F., Turbiner A.V., Winternitz P., Periodic orbits for an infinite family of classical superintegrable systems, J. Phys. A: Math. Theor., v.43, 015202, (2010).

[42] Tsiganov A.V., Duality between integrable Stäackel systems, J. Phys. A: Math. Gen., v.32(45), pp.7965-7982, (1999).

[43] Tsiganov A.V., Canonical transformations of the extended phase space, Toda lattices and the Stäackel family of integrable systems J. Phys. A: Math. Gen., v.33(2), pp. 4169-4182, (2000).

[44] Tsiganov A.V., On maximally superintegrable systems, Reg. Chaot. Dyn., v.13(3), pp.178190, (2008).

[45] Tsiganov A.V., Addition theorems and the Drach superintegrable systems, J. Phys. A: Math. Theor., v. 41, 335204, (2008).

[46] Tsiganov A.V., Leonard Euler: addition theorems and superintegrable systems, Reg. Chaot. Dyn., v.14(3), pp.389-406, (2009).

[47] Tsiganov A.V., On the superintegrable Richelot systems, J. Phys. A: Math. Theor. v. 43, 055201, 2010.

[48] Tsiganov A.V., Superintegrable Stäckel systems on the plane: elliptic and parabolic coordinates, SIGMA, v.8, 031, (2012).

[49] Tsiganov A.V., New bi-Hamiltonian systems on the plane, J. Math. Phys., v.58, 062901, 2017. 